\title{
EDITORIAL
}

\section{Necrotizing Enterocolitis: Finding Infants at Highest Risk}

\section{Journal of Perinatology (2008) 28, 655-656; doi:10.1038/jp.2008.134}

Despite decades of research, necrotizing enterocolitis (NEC) remains one of the conditions within our neonatal intensive care units with the highest mortality. Experience has garnered the common realization that preterm infants and those requiring surgical therapy will fare the worst. However, clear clinical predictors to identify positively which infants will progress with the disease remain elusive.

In this edition of The Journal of Perinatology, Moss and other investigators from six children's hospital across the United States present their data from their prospective, observational study. Although a negative study in which no new model could be developed to predict NEC, the analysis is an important contribution to further expand our knowledge of the risk factors for NEC progression. Dr Moss and colleagues characterized 455 infants with NEC into 'nonprogressors' who reached full feeding without surgical therapy or 'progressors' who either required surgical therapy or died from disease. Dedicated research nurses from the network collected data from a wide array of maternal, antenatal, intrapartum and newborn histories. Extensive daily feeding data including the mode and specific type of formula and/or total parenteral nutrition combination were also collected. Infants were followed during their inpatient hospitalization and extensive clinical data were collected relating to medications, radiology findings, ventilatory requirements, fluid and blood culture status. If infants received surgical therapy, surgical findings and postoperative data were also included in the analysis. The authors identified independent risk factors for progression of disease, with a particular focus on clinical information available at the time of diagnosis. Univariate analysis revealed NEC and progression to be associated with factors such as early gestational age, decreased birthweight, maternal age, intubation, compressions and portal venous gas, among others. Multivariate analysis identified 12 risk factors with 3 not previously described: teenaged mother, never have received enteral feeds before diagnosis and receipt of cardiac compressions/resuscitative drugs at delivery. Previously described risk factors were confirmed including factors such as Gramnegative bacteremia, portal venous gas, pneumatosis, metabolic acidosis at diagnosis, bandemia and low birth weight.

One of the most interesting findings of this paper is the progression of NEC in infants who had never received enteral feeds. One plausible explanation for this finding might be that infants with NEC were being diagnosed early in life and thus may have had less of an opportunity to start enteral feeds. However, to further validate the finding, the authors confirmed that the association of NEC progression with feeding was still maintained even when they controlled for age at diagnosis.

The authors also tried to develop a predictive model based on clinical factors at the time of diagnosis but the model performed poorly in discriminating which infants with NEC progressed $\left(R^{2}=0.46\right)$. Again, although unsuccessful in terms of development of a novel model to predict NEC progression, I believe the paper overall also reminds us to highlight several broad important points:

First, the study of neonatal conditions such as NEC is inherently challenging in that no single institution or practice has access to a large numbers of patients. On the other hand, the laws of statistics dictate that large patient numbers are prerequisite for valid and meaningful study. Thus, it is not surprising that some the of the best data to date elucidating the risk factors for NEC have been produced by large neonatal networks. ${ }^{1-6}$ These must be supported and will be vital as we move forward.

Second, to date we have not identified clear clinical predictors for infants with progressive NEC. The work under discussion that also could not develop a successful predictive model is the culmination of a tremendous effort of investigators and financial resources across multiple children's hospitals in the United States and is clearly one of the best efforts to date. Prospective studies ${ }^{7-14}$ including the one in this edition along with retrospective studies ${ }^{15,16}$ have failed to identify clinical factors that might allow us to identify narrowly infants at highest risk. Thus, it is clear that future efforts by these same networks must focus on genetic, proteomic and/or microbiologic investigations. These investigations taken together with clinical factors will be more likely to help delineate which infants are at the highest risk for NEC progression.

Finally, an appeal for improved analyses of surgical decisionmaking. Surgical therapy seems to be one of the final crashing points for this disease. A chain of events contributes to this final derailment that likely begins long before the surgeon arrives, a fact increasingly clear in that the specific type of operation for the disease does not seem to be centrally important. ${ }^{17,18}$ Nonetheless, I believe there is still more to be learned about surgical therapy such as factors relating to timing, role for abdominal decompression and so on. The decision whether to go ahead or hold off on surgical therapy is challenging enough to face at the moment it needs to be made. However, analyzing surgical decision-making after the fact is nearly impossible without comprehensive data. One of the 
impressive aspects of Dr Moss' network is the prospective gathering of data relating to surgical therapy and intraoperative findings, a practice that must be broadened to analyze an even wider array of factors relating to surgical decision-making and should be continued in future work.

\section{F Abdullah \\ Division of Pediatric Surgery, Johns Hopkins Medical Institutions, Baltimore, MD, USA E-mail:fa@jhmi.edu}

\section{References}

1 Fanaroff AA, Hack M, Walsh MC. The NICHD neonatal research network: changes in practice and outcomes during the first 15 years. Semin Perinatol 2003; 27: 281-287.

2 Guthrie SO, Gordon PV, Thomas V, Thorp JA, Peabody J, Clark RH. Necrotizing enterocolitis among neonates in the United States. J Perinatol 2003; 23: 278-285.

3 Horbar JD, Badger GJ, Carpenter JH, Fanaroff AV, Kilpatrick S, LaCorte M et al. Trends in mortality and morbidity for very low birth weight infants, 1991-1999. Pediatrics 2002; 110: $143-151$

4 Luig M, Lui K. Epidemiology of necrotizing enterocolitis - part I: changing regional trends in extremely preterm infants over 14 years. J Paediatr Child Health 2005; 41 : $169-173$.

5 Luig M, Lui K. Epidemiology of necrotizing enterocolitis - part II: risks and susceptibility of premature infants during the surfactant era: a regional study. J Paediatr Child Health 2005; 41: 174-179.

6 Sankaran K, Puckett B, Lee DS, Seshia M, Boulton J, Qiu Z et al. Variations in incidence of necrotizing enterocolitis in Canadian neonatal intensive care units. J Pediatr Gastroenterol Nutr 2004; 39: 366-372.

7 Berseth CL, Bisquera JA, Paje VU. Prolonging small feeding volumes early in life decreases the incidence of necrotizing enterocolitis in very low birth weight infants. Pediatrics 2003; 111: 529-534
8 Blakely ML, Lally KP, McDonald S, Brown RL, Barnhart D, Ricketts RR et al. Postoperative outcomes of extremely low birth-weight infants with necrotizing enterocolitis or isolated intestinal perforation: a prospective cohort study by the NICHD Neonatal Research Network. Ann Surg 2005; 241: 984-989.

9 Book LS, Herbst JJ, Atherton S0, Jung AL. Necrotizing enterocolitis in low-birth-weight infants fed an elemental formula. J Pediatr 1975; 87: 602-605.

10 Fast C, Rosegger H. Necrotizing enterocolitis prophylaxis: oral antibiotics and lyophilized enterobacteria vs oral immunoglobulins. Acta Paediatr Suppl 1994; 396 $86-90$.

11 Lin HC, Su BH, Chen AC, Lin T-W, Tsai C-H, Yeh T-F et al. Oral probiotics reduce the incidence and severity of necrotizing enterocolitis in very low birth weight infants. Pediatrics 2005; 115: $1-4$.

12 Lucas A, Cole TJ. Breast milk and neonatal necrotising enterocolitis. Lancet 1990; 336 $1519-1523$.

13 Ostertag SG, LaGamma EF, Reisen CE, Ferrentino FL. Early enteral feeding does not affect the incidence of necrotizing enterocolitis. Pediatrics 1986; 77 275-280.

14 Rayyis SF, Ambalavanan N, Wright L, Carlo WA. Randomized trial of 'slow' versus 'fast' feed advancements on the incidence of necrotizing enterocolitis in very low birth weight infants. J Pediatr 1999; 134: 293-297.

15 Llanos AR, Moss ME, Pinzon MC, Dye T, Sinkin RA, Kendig JW. Epidemiology of neonatal necrotising enterocolitis: a population-based study. Paediatr Perinat Epidemiol 2002; 16: 342-349.

16 Stoll BJ, Kanto WP Jr , Glass RI, Nahmias AJ, Brann Jr AW. Epidemiology of necrotizing enterocolitis: a case control study. J Pediatr 1980; 96 $447-451$.

17 Moss RL, Dimmitt RA, Barnhart DC, Sylvester KG, Brown RL, Powell DM et al. Laparotomy versus peritoneal drainage for necrotizing enterocolitis and perforation. $N$ Engl J Med 2006; 354: 2225-2234.

18 Blakely ML, Tyson JE, Lally KP, McDonald S, Stoll BJ, Stevenson DK et al. Laparotomy versus peritoneal drainage for necrotizing enterocolitis or isolated intestinal perforation in extremely low birth weight infants: outcomes through 18 months adjusted age. Pediatrics 2006; 117: e680-e687. 